\title{
Auf dem Weg zur objektiven Evaluation von Form, Volumen und Symmetrie in der Plastischen Chirurgie mittels intraoperativer 3D Scans
}

\author{
En Route for Objective Evaluation of Form, Volume, and Symmetry in Plastic \\ Surgery using 3-D Intraoperative Scans
}

Autoren

Institut

\section{K. C. Koban, T. Schenck, P. M. Metz, E. Volkmer, F. Haertnagl, V. Titze, R. E. Giunta}

Abteilung für Handchirurgie, Plastische Chirurgie und Ästhetische Chirurgie der Ludwig-Maximilians Universität München, Campus Innenstadt und Campus Großhadern

\author{
Schlüsselwörter \\ - 3-dimensionale \\ Fotodokumentation \\ - Operationsplanung \\ - Intraoperativer Scan \\ - Mobile 3D Scanner \\ - Volumetrie \\ Key words \\ - 3-dimensional \\ documentation \\ - operation planning \\ - intraoperative scanning \\ - mobile 3D scanning \\ - volumetry
}

eingereicht $\quad 3.3 .2016$

akzeptiert 7.3.2016

\section{Bibliografie}

DOI http://dx.doi.org/ 10.1055/s-0042-104506 Handchir Mikrochir Plast Chir 2016; 48: 78-84 (c) Georg Thieme Verlag KG Stuttgart · New York ISSN 0722-1819

\section{Korrespondenzadresse} Konstantin Christoph Koban Abteilung für Handchirurgie Plastische Chirurgie und Ästhetische Chirurgie Klinikum der LudwigMaximilians-Universität München

Pettenkoferstraße 8a 80336 München konstantin.koban@med. uni-muenchen.de

\section{Zusammenfassung \\ $\nabla$}

Hintergrund: Die 3-dimensionale (3D) Oberflächenerfassung durch Laserscanner und digitaler 3D Fotografie (Photogrammetrie) hat sich in den letzten Jahrzehnten stetig weiterentwickelt. Der Trend folgt ähnlich dem Smartphone Bereich der Maxime „kompakter, leichter, mobiler und komfortabler“. Obwohl 3D Scans in der Plastischen Chirurgie seit Ihren Anfängen in den 1980ern immer häufiger zur Beratung von Patienten und zur digitalen Dokumentation eingesetzt werden, so zeigte sich in den letzten Jahren keine einschneidende Entwicklung zur objektiven Unterstützung der Chirurgen für deren Eingriffe. Patienten, Material und Methoden: Unsere Arbeitsgruppe stellt eine neue Methode der intraoperativen 3D Erfassung von plastisch-rekonstruktiven Eingriffen anhand einer Auswahl von mobilen 3D Scanner Systemen vor. Diese könnten in Zukunft prä-, post-, als auch intraoperativ in der Befunderhebung, Therapiewahl und -Beratung, sowie Dokumentation den Operateur unterstützen.

Ergebnisse: Anhand mehrerer Patienten konnten die 3D Scanner erfolgreich für den intraoperativen Einsatz validiert werden. Insbesondere eine objektive, intraoperative Volumenmessung im Hinblick auf Form und Symmetrie wird dadurch möglich.

Schlussfolgerung: Anhand dieser Arbeit teilen wir unsere ersten Erfahrungen in der Anwendung neuer mobiler 3D Kamera Systeme, diskutieren Vor- und Nachteile und präsentieren Beispiele des intraoperativen Einsatzes.

\section{Einleitung \\ $\nabla$}

Schon seit der Antike bestand das Bestreben die physiologischen und pathophysiologischen Veränderungen des Körpers zu Vermessen und Objektivieren. Obwohl lineare Messverfahren und Umfangsmessungen bereits bekannt waren, zeigte

\section{Abstract \\ $\nabla$}

Background: There has been ongoing development in the field of 3-dimensional (3-D) Surface Imaging by laser scanner and digital 3-D photography (Photogrammetry) in recent years. Manufacturers tend to make new 3-D cameras compact, light-weighted, mobile, and user-friendly, similar to the development on the smartphone market. Although 3D scans have been used for patient consultations and digital documentation in Plastic Surgery since the 1980, there has been no significant development lately regarding its use for objective assistance during surgery.

Patients, Material and Methods: Our research team presents a new intraoperative 3-D scanning method for plastic-surgical procedures with selected mobile 3-D scanner systems. In the future, these might assist surgeons with the pre-, post-, and intraoperative 3-D analysis, choice of therapy, consultation, and documentation.

Results: The 3-D scanners were successfully validated for their intraoperative application to several patients. With their introduction, an intraoperative, objective measurement of volume to evaluate form and symmetry was possible.

Conclusion: In this work, we share our first experience with the intraoperative use of new mobile 3D camera systems, discuss pros and cons, and show selected patient examples. 

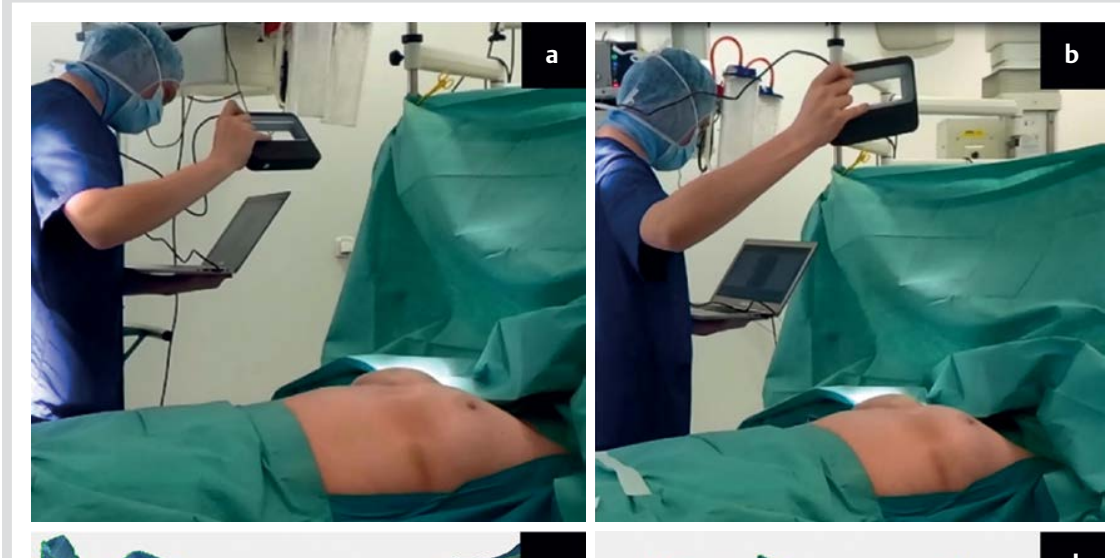

Abb. 1 Beispiel 1: Ansicht der intraoperativen Brustaufnahme a, b mit dem Sense Scanner bei einer 44-jährigen Patientin vor Mammaaugmentation. Kritisch sind die manuelle Ausführung und das Fokussieren auf einen Laptop anstelle des Scanners. 3D-Aufnahmen vor $\mathbf{c}, \mathbf{d}$ und nach $\mathbf{e}, \mathbf{f}$ submuskulärer Mammaaugmentation mittels inframammären Zugang und $375 \mathrm{~mL}$ anatomischen Implantaten.
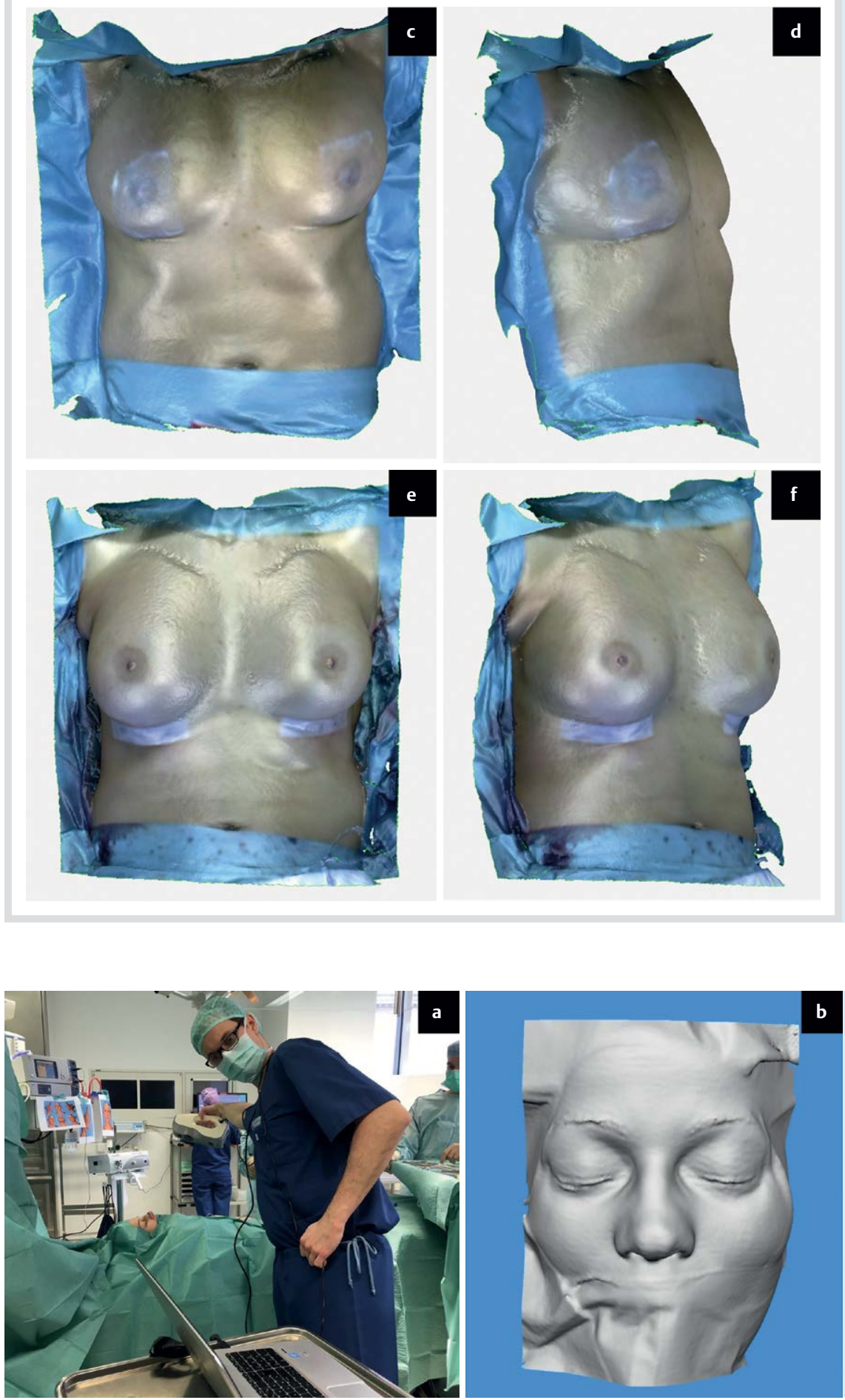

Abb. 2 Beispiel 2: Intraoperative Gesichtsaufnahme einer 37-jährigen Patientin vor angleichender Rhinoplastik bei Sattelnase. a Ebenfalls ist die Fokussierung auf den Computer während des Scans erkenntlich. b Intraoperative Aufnahme hier ohne Textur des abgedeckten Gesichts. Feinste Details wie Augenbrauen werden akkurat erfasst. 

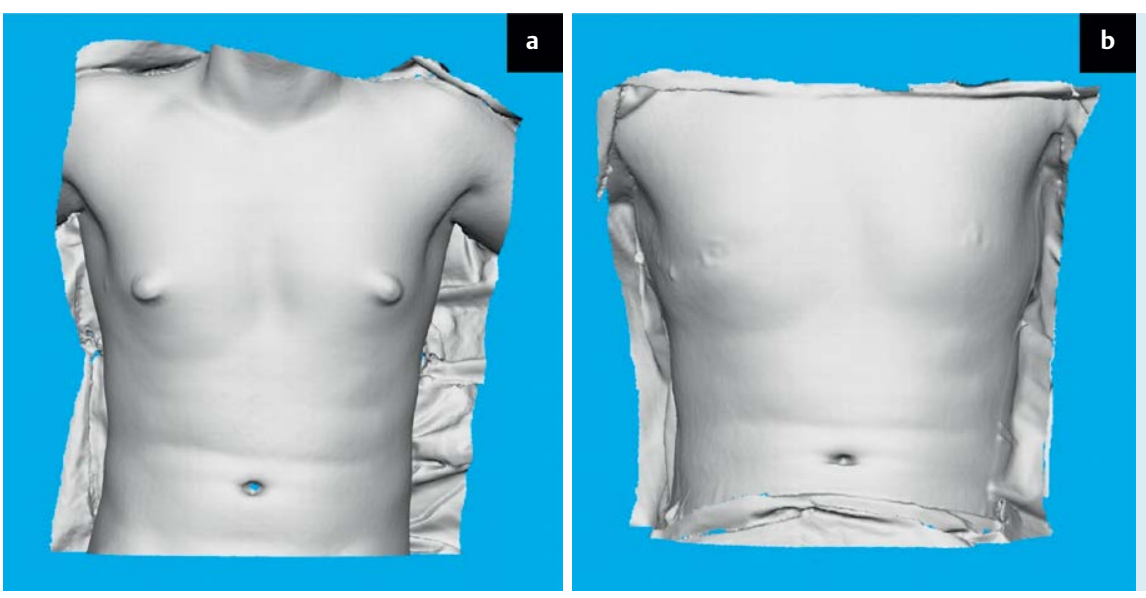

Abb. 3 Beispiel 3: 24-jähriger Patient mit Gynäkomastie $2^{\circ}$. Intraoperative Aufnahme mittels Artec Eva vor a und nach Liposuktion und subkutaner Mastektomie $\mathbf{b}$. Insgesamt wurden beidseits $250 \mathrm{~mL}$ Lipoaspirat abgesaugt, sowie $23 \mathrm{~mL}$ Drüsengewebe links und $37 \mathrm{~mL}$ rechts entfernt.

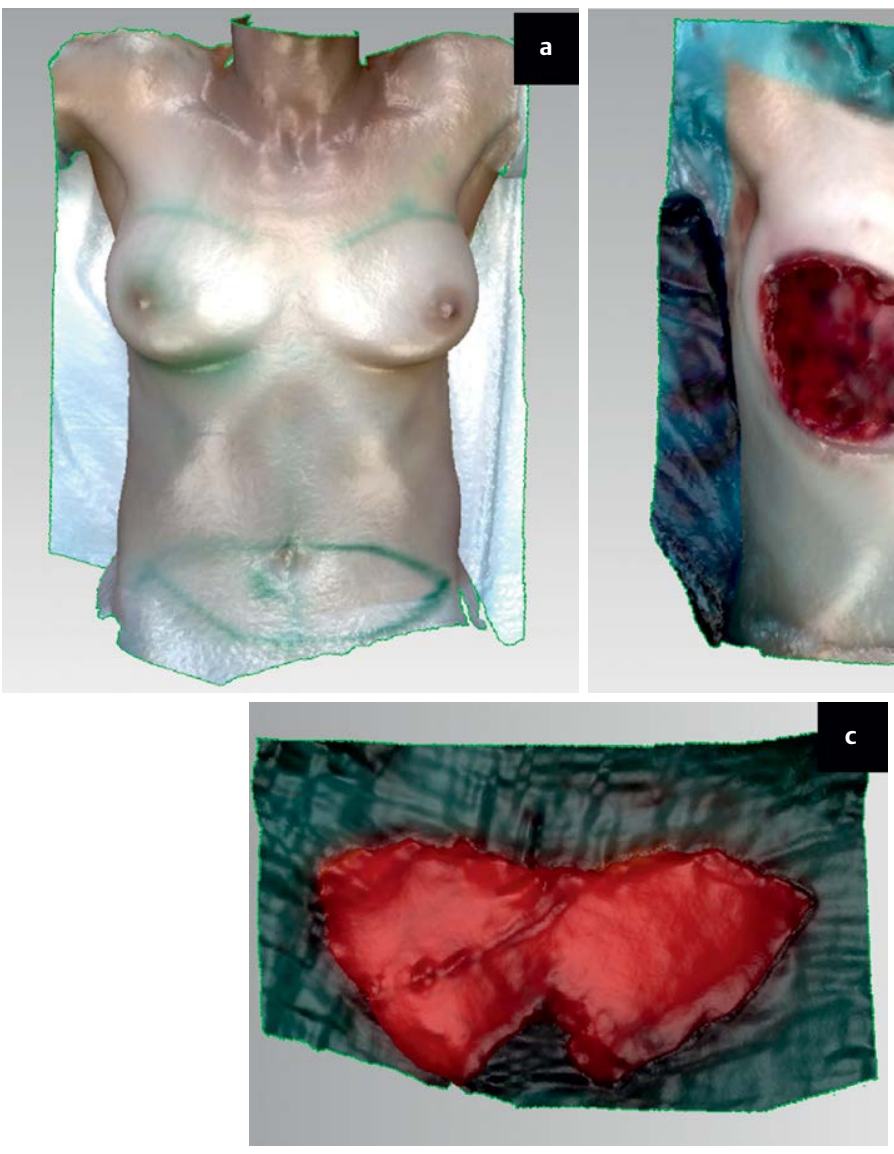

Abb. 4 Beispiel 4: Intraoperative 3-D-Aufnahmen der Sense-Kamera einer 51-jährigen Patientin mit zentralem T1b Mammakarzinom in der rechten Brust vor einzeitiger Mastektomie und Rekonstruktion. Hier zu sehen sind der Befund präoperativ auf OP-Liege mit Anzeichnung der klassischen Brustmaße und der Lappenplastik im Bauchbereich a, nach Mastektomie $\mathbf{b}$, sowie der gehobene DIEP-Lappenplastik mit Arterie c vor dem Anschluss im Brustbereich. Im Vergleich zu den Vectra Aufnahmen zeigt sich hier ein deutlicher Verlust der Texturqualität.

Proportionen messbar gemacht werden [1]. Jedoch produzieren solche Fotos häufig aufgrund eingeschränkter Technik, fehlerhafter Patienten Positionierung und Kameraführung nur unzureichend standardisierte Aufnahmen und damit fehlerhafte Messungen ( $\bullet$ Abb. 1-4).

Neuartige Möglichkeiten eröffneten sich in den letzten Jahrzehnten durch die Einführung von Technologien zur digitalen 3D Oberflächenerfassung von Körpern [1,2]. Diese zeichnen sich durch eine schnelle Datenakquise im Vergleich zu Distanz- und Umfangsmessungen, Wasserverdrängung [3] und anderen herkömmlichen Verfahren aus. Ein hohes Potenzial dieser Technik zeigt sich auch in der Plastischen Chirurgie zur Wiederherstellung oder Verbesserung gestörter Funktion und Form des Körpers, da das behandelte Körperareal oft unmittelbar durch den behandelnden Chirurgen intraoperativ beurteilbar werden muss.
Bei der Erstellung eines digitalen 3D Oberflächenmodells einer Körperregion mittels Photogrammetrie handelt es sich um eine 3D Rekonstruktion aus Fotos des Objekts. Neben den ursprünglich genutzten Laserscannern hat sich dieses Verfahren in den letzten 10 Jahren zur Dokumentation im Bereich der Plastischen Chirurgie etabliert und anhand zahlreicher Studien [4] wurden überwiegend große, statische 3D Kamera Systemen zur Dokumentation von Eingriffen evaluiert [5], die prä- und postoperativ eine Evaluation erlauben.

Im Rahmen der Digitalisierung des Klinikalltages und der Weiterentwicklung vor allem im Smartphone Bereichs zeigte sich eine immer weitere Komprimierung solcher Systeme zu kleinen, handgeführten 3D Kameras und Scanner, welche zuletzt bis auf die Größe einer Smartphone Erweiterung reduziert wurden oder gar nur als Software-Lösung [6] eingesetzt werden können. 
Tab. 1 Mobile 3D Kameras für den intraoperativen Einsatz.

\begin{tabular}{|c|c|c|c|c|}
\hline & Vectra XT & Artec EVA & Sense & iSense \\
\hline $\begin{array}{l}\text { Technik und Auf- } \\
\text { nahmeprinzip }\end{array}$ & $\begin{array}{l}\text { 3D digitale Fotografie } \\
\text { (Photogrammetrie) } \\
\text { - Einzeitiges Foto }\end{array}$ & $\begin{array}{l}\text { Structured Light“ } \\
\text { Prinzip }{ }^{1} \\
\text { - Scannen }\end{array}$ & $\begin{array}{l}\text { Infrarot Tiefensensor+Kamera } \\
\text { - Scannen }\end{array}$ & $\begin{array}{l}\text { Infrarot Tiefensensor+Kamera } \\
\text { - Scannen }\end{array}$ \\
\hline Aufnahmegröße & Einmalig $180^{\circ}$ & Bis zu $360^{\circ}$ & Bis zu $360^{\circ}$ & Bis zu $360^{\circ}$ \\
\hline Aufnahmebereich & $\begin{array}{l}\text { Hals \& Gesicht, Torso, } \\
\text { Gluteal }\end{array}$ & Kopf bis Fuß & $\begin{array}{l}\text { Hals \& Gesicht, Torso, Gluteal } \\
\text { Arme, Beine }\end{array}$ & Hals \& Gesicht, Torso, Gluteal Arme, Beine \\
\hline Aufnahmezeit & $2 \mathrm{~ms}$ & 10-60 s (bis zu $30 \mathrm{fps)}$ & $15-60$ s (bis zu $30 \mathrm{fps}$ ) & 15-60 s (bis zu 30 fps) \\
\hline Verarbeitungszeit & $120 \mathrm{~s}$ & $300 \mathrm{~s}$ & $60 \mathrm{~s}$ & $60 \mathrm{~s}$ \\
\hline Abweichung & & $<0.1 \mathrm{~mm} \mathrm{RMS}$ & $0.1-3 \mathrm{~mm}$ RMS & $0.1-3 \mathrm{~mm}$ RMS \\
\hline Peripherie & $\begin{array}{l}\text { Leistungsstarker } \\
\text { Computer }\end{array}$ & $\begin{array}{l}\text { Leistungsstarker } \\
\text { Computer/Laptop }\end{array}$ & Verbraucher Laptop & Apple iPad der 3. Generation aufwärts \\
\hline
\end{tabular}

Diese 3D Modelle können sowohl zu Dokumentationszwecken als auch zur Datenanalyse und OP-Planung [7] dient. In heutigen Hybrid-Operationssälen sind bereits erweiterte Datensätze von 3D-CTs [8] und -MRTS integriert und für die Operateure z.B. in der Leberchirurgie und Strahlentherapie verwendbar. Dadurch wurden schwierige Operationsschritte besser planbar, die Orientierung im komplexen 3-dimensionalen Raum erleichtert und die Strahlendosis besser fokussierbar.

In diesem Rahmen wäre eine ergänzende nicht-invasive, strahlungsfreie Diagnostik, wie es die berührungslose 3-dimensionale Vermessung von Oberflächen durch 3D Kameras bietet, von Vorteil. Bislang war es mit den vorhandenen Systemen jedoch nicht möglich während einer Operation objektive Daten zu Form, Volumen und Symmetrie dem Operateur zu visualisieren und dadurch die Operateure zu unterstützen, obwohl ein möglicher Nutzen bereits beschrieben wurde $[9,10]$.

Ziel dieser Arbeit ist die erste Erprobung neuer mobiler 3D Kamerasysteme für intraoperative Aufnahmen und Evaluierung möglicher Einsatzgebiete. Unsere Arbeitsgruppe stellt hierbei eine neue Methode der intraoperativen 3D Erfassung von plastisch-chirurgischen Eingriffen vor, welche in Zukunft die prä-, post-, als auch intraoperative Befunderhebung, Therapiewahl und -Beratung, sowie Dokumentation den Operateur zu jedem Zeitpunkt ermöglichen könnte.

\section{Patienten, Material und Methoden \\ $\nabla$}

\section{Manuelle intraoperative Messmethoden}

Zu den häufigsten Messmethoden im Rahmen plastisch-chirurgischer Operationen gehören die manuelle Maßbandmessung und Winkelmessungen. Hierbei ist eine präoperative Anzeichnung mit Ausmessung an den Patienten im Stehen für fast alle Operationen essentiell $[11,12]$. Intraoperative Änderungen werden immer mit den zuvor erfassten Messdaten verglichen und angepasst.

Im Rahmen von Eingriffen in der Brust wie der Mastektomie oder Abdomen bei Abdominoplastik werden häufig auch zur symmetrischen Angleichung dieser das Gewicht der Resektate gewogen oder das Volumen mittels Wasserverdrängung bestimmt. Bei Wasser-assistierter Liposuktion und Lipofilling ist ebenfalls ein korrektes Erfassen von injizierter Tumeszenzlösung, abgesaugtem Lipoaspirat und eingebrachten Lipofiltrat von eminenter Bedeutung.

\section{D intraoperative Messungen}

Wie bereits durch Tzou C. et al. [4] zusammengefasst, ermöglicht die 3D Oberflächenerfassung reproduzierbare Messungen von Distanzen, Umfängen, Winkeln und Proportionen. Auch konnte die Veränderung bei brustchirurgischen Eingriffen mittels 3D Scans auch im Vergleich zum MRT evaluiert werden [13].

Eine Erhebung digitaler Messwerte im Bereich der Brust sind z. B. die klassischen Brustmaße $[\mathrm{cm}]$, die Brustoberflächen $\left[\mathrm{cm}^{2}\right]$, Brustvolumenmessungen $\left[\mathrm{cm}^{3}\right]$ und Brustsymmetrieevaluation $[14,15]$.

\section{Anforderungen an mobile 3D Scanner}

Aktuelle Systeme müssen für den intraoperativen Einsatz eine zuverlässige 3-D Beurteilung von Form, Kontur, Symmetrie und Textur des Körpers ermöglichen.

Wir erarbeiteten aus der aktuellen Literatur [16] und klinischen Erfahrung Anforderungen für intraoperative, mobile 3D Aufnahmen, welche gegenwärtige Scanner Systeme erfüllen müssen. Eine Auflistung der resultierenden Auswahl für 3D Kameras und Scanner anhand der zuvor getroffenen Parameter spiegelt $\bullet$ Tab. 1 dar. Zum intraoperativen Einsatz in der Plastischen Chirurgie wurden die mobilen Scanner Sense (3D Systems) und Artec Eva (Artec Inc.) eingesetzt. Für alle Geräte liegt eine Zertifizierung nach CE-Norm vor.

Der Sense 3D Scanner (3D SYSTEMS ${ }^{\circledR}$ ) ist eine handgeführter 3D Kamera (400g). Anhand von Tiefeninformation-erfassenden Infrarotsensoren und einer eingebauten Kamera wird ein Objekt 3-dimensional mit bis zu 30 Bildern pro Sekunde erfasst. Das Gerät wird zur Berechnung des 3D Modells mit einem Computer oder Tablet verbunden. Durch diese Kombination ist die Kamera leicht im Raum um das Objekt zu navigieren. Der Aufnahmeprozess erinnerte am ehesten an den einer Videokamera wie in späteren Beispielen dargestellt.

Bei der ebenfalls handlichen Artec Eva (Artec Inc.) handelt es sich um eine vor allem industriell eingesetzten 3D Kamera $(800 \mathrm{~g})$, welche hochauflösende 3D Modelle in Echtzeit anhand eines „Structured Light“ Verfahrens durch Lichtblitze bei bis zu 30 Bildern pro Sekunde an einem leistungsstarken Laptop erfasst. Das Gerät funktioniert nur durch eine eigene, permanente Stromanbindung.

Als Kontrast wird in unserer Abteilung das etablierte Vectra Volumetric 3D Surface Imaging System (Canfield Scientific ${ }^{\circledR}, \mathrm{NJ}$, USA) als große, statische 3D Kamera zur Dokumentation von Eingriffen eingesetzt. Das große, fest installierte Kamerasystem dient nur als Referenz für die mobilen Systeme am stehenden Patienten. 


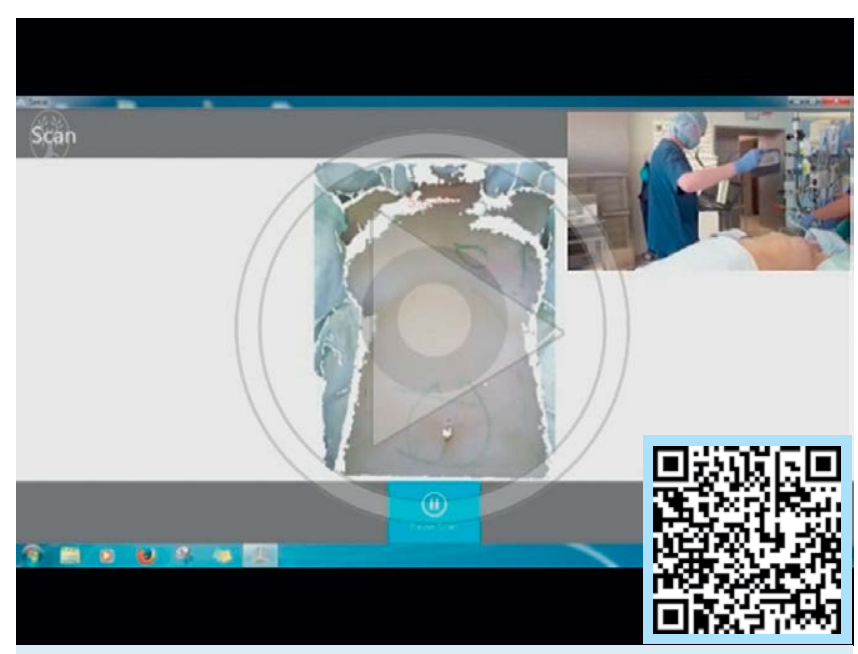

Video 1 Video der Sense 3D-Erfassung einer weiteren Patientin auf dem Operationstisch mit Bild in Bild Darstellung des Scanvorgangs durch den Untersuchers.

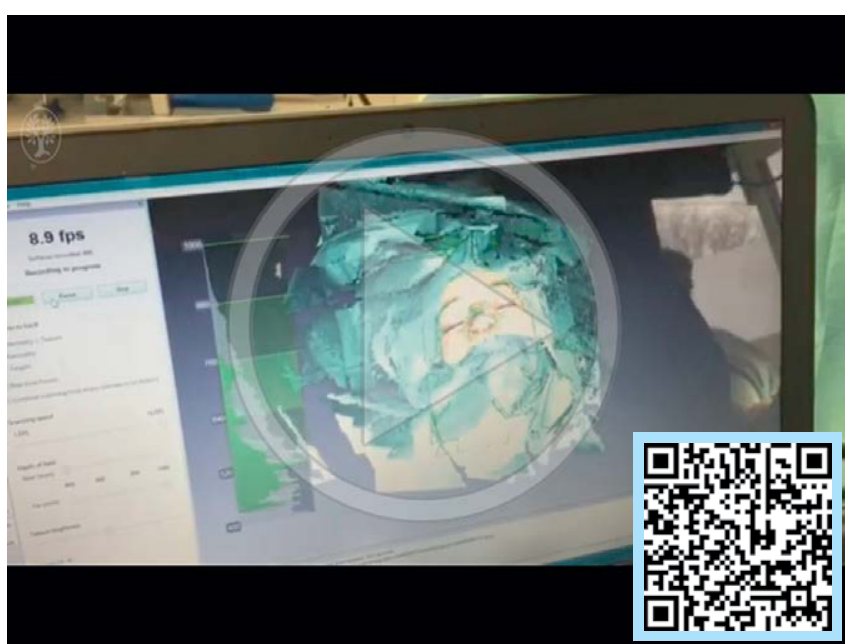

Video 2 Intraoperatives Video der Artec Eva 3D-Erfassung einer 37-jährigen Patientin vor angleichender Rhinoplastik bei Sattelnase. Zu sehen ist die Darstellung für den Untersucher und die Generierung des 3D Modells.

\section{Aufnahmetechnik}

Illustrierend die Aufnahmetechnik des Sense Scanners anhand einer beidseitigen Mammaaugmentation (Beispiel 1). Das Gerät wird von einer Seite über die Mitte zur anderen Seite im ZickZack-Muster geführt. Der Computer zeigt fehlende, noch nicht erfasste Bereiche an und gibt auch bei Abweichung vom Scan Kurs eine Fehlermeldung mit korrigierendem Tracking an.

- Abb. 2 und 3 zeigen die Darstellung für den Artec Eva Scanner. Begleitend ein Video der 3D Aufnahme bei einer weiteren Patientin ('미 Video 1, 2).

\section{Einsatzmöglichkeiten mobiler Scanner}

Form, Kontur und Symmetrie und des Körpers werden maßgeblich während der Operation festgelegt und unterliegen dem subjektiven Eindruck des oder der Operateure. Eine Übersicht der potentiellen Einsatzmöglichkeiten gibt $\bullet$ Tab. 2.

Zu den Aufgaben der Plastischen Chirurgie gehört die Rekonstruktion großer Gewebedefekte an Körperstamm und Extremitäten mit freien Gewebetransplantaten anderer Körperregionen. Hier zu nennen ist insbesondere für circa 20000 Brustkrebs-
Tab. 2 Einsatzmöglichkeiten mobiler Scanner in der Plastischen Chirurgie.

\begin{tabular}{|c|c|}
\hline $\begin{array}{l}\text { Plastische } \\
\text { Chirurgie }\end{array}$ & Brust-, Gesichts- und Nasenchirurgie \\
\hline & $\begin{array}{l}\text { Symmetrie- und Formbewertung } \\
\text { Volumenmessungen } \\
\text { Lappenplastiken } \\
\text { Lymphödem: Therapiekontrolle, Verlauf } \\
\text { Liposuktion und Lipofilling } \\
\text { Gebrauch von dermalen Füllersubstanzen, Botolinumtoxin } \\
\text { Wundheilung }\end{array}$ \\
\hline \multicolumn{2}{|c|}{$\begin{array}{l}\text { Verbrennungs- } \\
\text { chirurgie }\end{array}$} \\
\hline & $\begin{array}{l}\text { Verbrennungen der Körperoberfläche (\% KOF) } \\
\text { Berechnung von Defektarealen zur Deckung } \\
\text { Verlaufskontrollen in 3D }\end{array}$ \\
\hline \multicolumn{2}{|c|}{ Handchirurgie } \\
\hline & $\begin{array}{l}\text { Beurteilung Schwellungsverläufe nach Eingriffen an der Hand } \\
\text { Verbinden von 3D-CT Aufnahmen mit mobilen Scans }\end{array}$ \\
\hline \multicolumn{2}{|c|}{ Tab. 3 Anforderungen an Scanner für den klinischen Einsatz. } \\
\hline \multicolumn{2}{|c|}{$\begin{array}{l}\text { - Auflösung } \\
\text { - Größe/Handling } \\
\text { - Aufnahmedauer (Akquisitionszeit) } \\
\text { - Softwaremöglichkeiten } \\
\text { - Geschwindigkeit der Datenanalyse } \\
\text { - Benutzerfreundlichkeit } \\
\text { - Reproduzierbarkeit } \\
\text { - Einsatzmöglichkeit } \\
\text { - Farbaufnahmen } \\
\text { - Kosten }\end{array}$} \\
\hline
\end{tabular}

patienten nach kompletter Brustresektion die Rekonstruktion mittels freier Gewebetransplantation vom Unterbauch (DIEPLappenplastik, ○ Abb. 4). Herausfordernd ist neben der aufwändigen Durchführung der Operation insbesondere die korrekte Planung der Operation, um vorausschauend per Überkorrektur der operierten Seite eine Angleichung zur gesunden Brust im Verlauf zu erreichen. Im Regelfall wünschen Patienten häufig postoperativ eine weitere Korrektur- und Symmetrieanpassung was mehrere Folgeoperationen nach sich ziehen kann ( $\bullet$ Tab. 3).

\section{Diskussion}

Mit dem Erscheinen immer kompakterer, mobiler und erschwinglicherer 3-D Bilderfassungsgeräte ist eine erweiterte Einsatzmöglichkeit auch für den intraoperativen Einsatz gegeben. Von Vorteil der mobilen Systeme ist, dass sie flexibel in jeder Umgebung wie einem Operationssaal eingesetzt werden können und zudem auch bei immobilen Patienten eine Aufnahme gewährleisten.

Ebenfalls sind anhand des intraoperative Einsatzes am Patienten ein geeigneter Versuchsablauf sowie die Evaluierung benötigter Daten für den Operateur zu evaluieren.

Laserscan Systeme $[13,17,18]$ bieten die höchstmögliche Präzision eines 3D Scans durch punktuelle Abtastung der Oberfläche. Trotz der großen Genauigkeit steht der breiten klinischen Anwendung die Nachteile der Kosten eines Laserscanners und der verlängerten Aufnahmedauer durch mehrfache Scans für $360^{\circ}$ Aufnahmen entgegen. Ein intraoperativer Einsatz wäre nur mit hohem Aufwand möglich. 
Im Vergleich lassen sich großflächige 3D Aufnahmen durch die hier beschriebenen Systeme in weniger als 30 Sekunden herstellen, wobei es zur Millisekunden-schnellen Aufnahme des Vectra Gerätes, welches Bewegungsartefakte eliminiert, die längere Akquisitionszeit beider Geräte Sense als auch Artec Eva ein Kritikpunkt darstellt und Fehler der Volumenberechnung durch Bewegungsartefakte begünstigen kann.

Obwohl wir für beide Geräte in den dargestellten Beispielen nur äußerst geringe Abweichungen gegenüber dem Vectra System finden konnten, so zeigte sich insbesondere für den Sense Scanner nur eine unbefriedigende Farbqualität der Aufnahmen. Das Sense System lässt zusätzlich insbesondere bei mannigfaltigen Oberflächen im Rahmen von Adipositas und feinen Details wie im Gesichtsbereich Limitierungen erkennen. Weitergehende Untersuchungen an einem großen Patientenkontingent und an verschiedenen Anwendungen sind jedoch für eine detailliertere Bewertung der neuen Methode dringend nötig.

Mit dem Vectra System als Referenz ist zu beachten, dass für reproduzierbare Ergebnisse neben der konstanten Positionierung des Patienten vor dem Scanner vor allem dessen Körperhaltung auf dem Operationstisch zu beachten sind $[12,19]$. Mit unterschiedlicher Lagerung oder Armhaltung ändern sich in den gemessen Regionen die für das Verfahren ausschlaggebenden Maße der Oberflächenkonturen derart, dass eine sinnvolle Auswertung im Vergleich zu späteren Aufnahmen unmöglich werden kann $[20,21]$. Dementsprechend sind Standards für reproduzierbare Aufnahmeeinstellungen verschiedener Körperregionen zu erstellen, welche die Grundlage für den intraoperativen Einsatz bilden.

Mit dem Erscheinen immer kompakterer, mobiler und erschwinglicherer 3-D Bilderfassungsgeräte [22] ist eine erweiterte Einsatzmöglichkeit für den intraoperativen Einsatz auch in anderen Settings in naher Zukunft vorstellbar.

\section{Ausblick}

$\nabla$

Die Schwelle der 3D Visualisierung und Quantifizierung in die klinische Translation ist in den letzten Jahren übertreten worden. Damit ist eine Vielzahl an möglichen Anwendungen umsetzbar und es sind neue Studien mit klinische Daten in den nächsten Jahren zu erwarten.

Wir konnten anhand erster Beispiele neue 3D Kamerasysteme im intraoperativen Einsatz mit vielversprechenden Ergebnissen erfolgreich einsetzen.

Im Rahmen der Translation der Verfahren sind zu erwartende und unerwartete Hürden und Limitationen durch detaillierte Validierung verschiedener Einsatzmöglichkeiten an größeren Patientenkollektiven notwendig. Ein Erschwernis ist zu gegenwärtigen Zeitpunkt, dass die Patienten Untersuchung, Beratung und Planung der Patienten am stehenden Patienten in Untersuchungsräumen stattfindet, wohingegen die Operationen am liegenden Patienten im OP stattfindet und der Patient z.B. in Brustchirurgie allenfalls intraoperativ aufgesetzt werden kann [12].

Insbesondere im Rahmen der Evidenzbasierten Medizin könnte eine reproduzierbare und individuelle Planung vieler rekonstruktiver Eingriffe auch außerhalb der Plastischen Chirurgie ermöglicht werden und damit eine wesentliche Optimierung im Vergleich zum derzeitig üblichen „Augenmaß“ des Operateurs erreicht werden.
Hierbei könnte ein voll ausgereiftes 3D Kamerasystem für die patienten- und eingriffsspezifische Dokumentation, individuelle Planung einer Operation, intraoperative Unterstützung des Operateurs und postoperative Verlaufskontrollen eingesetzt werden. Wir sehen ein hohes Potenzial darin, Chirurgen mit objektiven Parametern durch den Einsatz neuer mobiler Geräte zu unterstützen, sowie eine reproduzierbare Entscheidungsfindung aufgrund einer zusätzlichen objektiven Bewertung außerhalb des Operationssaals zu ermöglichen. In den letzten Jahren begannen Simulationen von Eingriffen $[7,10,23]$ größeren Einzug in das operative Geschehen bei rekonstruktiven Eingriffen zu erhalten. Als Nachteil der 3D Techniken ist der für hochwertige Scanner derzeit noch hohe Preis der Hard und Software zu sehen. Es ist jedoch davon auszugehen, dass die Preise zunehmend sinken werden.

\section{Danksagung}

$\nabla$

Besonderer Dank gilt dem Förderprogramm für Forschung und Lehre (FöFoLe) der Ludwig-Maximilians-Universität, welches einen Teil dieser Arbeit ermöglichte (Projekt Nr. 843).

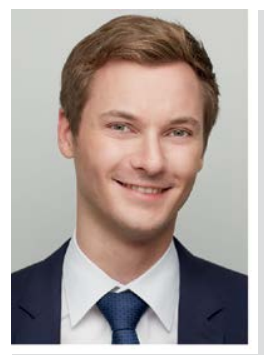

\section{Konstantin Koban}

geb. 1989 in Berlin. Studium der Humanmedizin an der Ludwig-Maximilians-Universität München mit Studienaufenthalten in London und Berlin. Dissertation zur „3D Oberflächenerfassung im klinischen Alltag" unter Prof. Giunta an der LMU Universität. Seit 2015 Assistenzarzt im Klinikum der Universität München in der Abteilung für Handchirurgie, Plastische Chirurgie und Ästhetische Chirurgie (Prof. Giunta).

Interessenkonflikt: Nein

\section{Literatur}

1 Jacobs RA. Three-dimensional photography. Plast Reconstr Surg 2001; 107: 276-277

2 Losken A, Seify H, Denson DD et al. Validating three-dimensional imaging of the breast. Ann Plast Surg 2005; 54: 471-476 discussion 477-478

3 Yip JM, Mouratova N, Jeffery RM et al. Accurate assessment of breast volume: a study comparing the volumetric gold standard (direct water displacement measurement of mastectomy specimen) with a 3D laser scanning technique. Ann Plast Surg 2012; 68: 135-141

4 Tzou CH, Artner NM, Pona I et al. Comparison of three-dimensional surface-imaging systems. J Plast Reconstr Aesthet Surg 2014; 67: 489-497

5 Spanholtz TA, Leitsch S, Holzbach T et al. 3-dimensional imaging systems: first experience in planning and documentation of plastic surgery procedures. Handchir Mikrochir Plast Chir 2012; 44: 234-239

6 Koban KC, Leitsch S, Holzbach T et al. 3D-imaging and analysis for plastic surgery by smartphone and tablet: an alternative to professional systems? Handchir Mikrochir Plast Chir 2014; 46: 97-104

7 Lekakis G, Claes P, Hamilton GS $3^{\text {rd }}$ et al. Three-Dimensional Surface Imaging and the Continuous Evolution of Preoperative and Postoperative Assessment in Rhinoplasty. Facial Plast Surg 2016; 32: 88-94

8 Khambay B, Nebel JC, Bowman J et al. 3D stereophotogrammetric image superimposition onto 3D CT scan images: the future of orthognathic surgery. A pilot study. Int J Adult Orthodon Orthognath Surg 2002; 17: $331-341$ 
9 Nahabedian MY, Galdino G. Symmetrical breast reconstruction: is there a role for three-dimensional digital photography? Plast Reconstr Surg 2003; 112: 1582-1590

10 Eder $M$, Grabhorn A, Waldenfels $F$ et al. Prediction of breast resection weight in reduction mammaplasty based on 3-dimensional surface imaging. Surg Innov 2013; 20: 356-364

11 Catanuto G, Patete P, Spano A et al. New technologies for the assessment of breast surgical outcomes. Aesthet Surg J 2009; 29: 505-508

12 Khatam H, Reece GP, Fingeret MC et al. In-vivo quantification of human breast deformation associated with the position change from supine to upright. Med Eng Phys 2015; 37: 13-22

13 Eder M, Schneider A, Feussner $H$ et al. Breast volume assessment based on 3D surface geometry: verification of the method using MR imaging. Biomed Tech (Berl) 2008; 53: 112-121

14 Henseler H, Khambay B, Ju X et al. Landmark-based statistical procrustes analysis in the examination of breast shape and symmetry. Handchir Mikrochir Plast Chir 2014; 46: 342-349

15 Eder $M$, Waldenfels FV, Swobodnik A et al. Objective breast symmetry evaluation using 3-D surface imaging. Breast 2012; 21: 152-158

$16 X i W$, Perdanasari AT, Ong Y et al. Objective breast volume, shape and surface area assessment: a systematic review of breast measurement methods. Aesthetic Plast Surg 2014; 38: 1116-1130
17 Kovacs L, Eder M, Papadopulos NA et al. Validating 3-dimensional imaging of the breast. Ann Plast Surg 2005; 55: 695-696

18 Kovacs L, Zimmermann A, Brockmann G et al. Accuracy and precision of the three-dimensional assessment of the facial surface using a 3-D laser scanner. IEEE Trans Med Imaging 2006; 25: 742-754

19 Maal TJ, Verhamme LM, van Loon B et al. Variation of the face in rest using 3D stereophotogrammetry. Int J Oral Maxillofac Surg 2011; 40: $1252-1257$

20 Liu C, Ji K, Sun J et al. Does respiration influence breast volumetric change measurement with the three-dimensional scanning technique? Aesthetic Plast Surg 2014; 38: 115-119

21 Henseler $H, J u X$, Ayoub $A$ et al. The importance of the pose in threedimensional imaging of the ptotic breast. J Plast Reconstr Aesthet Surg 2013; 66: 1551-1556

22 Wheat JS, Choppin S, Goyal A. Development and assessment of a Microsoft Kinect based system for imaging the breast in three dimensions. Med Eng Phys 2014 DOI: 10.1016/j.medengphy.2013.12.018

23 Roostaeian J, Adams WP Jr. Three-Dimensional Imaging for Breast Augmentation: Is This Technology Providing Accurate Simulations? Aesthet Surg J 2014; 34: 857-875 